\title{
Cricotracheal Resection in a Patient with Severe Subglottic Stenosis - Advantages of a Temporary Non-Cannulated Tracheostomy
}

\author{
Todor Miroslavov Popov1, Tzvetomir Marinov², Julian Rangachev1, Dimitar Konov1, Maya Belitova2 \\ ${ }^{1}$ Department of Otolaryngology, Medical University of Sofia, Sofia, Bulgaria \\ ${ }^{2}$ Department of Anesthesiology and Intensive Care, Medical University of Sofia, Sofia, Bulgaria
}

Background: Stenosis in the area of the cricotracheal segment is still a challenging problem to be dealt with. Post-intubational cases mark an increase in recent years due to the advances in intensive care, thoracic surgery and neurosurgery departments.

Case Report: This paper describes a case report of a patient with severe subglottic stenosis (grade III according to the Cotton-Myer scale), introduces a new option in cricotracheal resections - postoperative temporary non-cannulated tracheostomy and describes its advantages.

Conclusion: This variation of classical operative techniques provides additional fixation of the trachea, thus relieving any transitory tension on the anastomosis; acts as a valve and decreases the air-pressure in the upper airways during coughing and sneezing in the postoperative period; and is an easy access point for video tracheoscopy of the anastomosis.

Keywords: Cricotracheal resection, stenosis, trachea, larynx, tracheostomy
Stenosis in the area of the cricotracheal segment is still a challenging problem to be dealt with. Post-intubational cases mark an increase in recent years due to the advances in intensive care, thoracic surgery and neurosurgery departments (1). Pioneers such as Pearson, Grillo and Perelman have set the classical principles of this type of surgery (2), but new techniques and ideas are still being introduced in clinical practice $(3,4)$.

\section{CASE PRESENTATION}

\section{Patient preoperative assessment}

A 35-year old male patient was admitted to the Department of Otorhinolaryngology with a compensated inspiratory dyspnea and biphasic stridor. Video fibroscopy revealed a severe subglottic stenosis grade III according to the Cotton-
Myer scale. Computed tomography scan of the neck and chest confirmed an hourglass-shaped subglottic stenosis which carried down to the second tracheal ring (Figure 1). Three months prior to hospitalization, the patient underwent aortic valve replacement because of severe regurgitation after bacterial endocarditis. The patient was intubated in an intensive care unit for 5 days. A month after this procedure, the first signs of breathing problems began to appear. The cardiac status before our intervention displayed good functionality of the replaced valve but moderate insufficiency of the mitral and tricuspid valves, with an ejection fraction of $28 \%$. The patient showed a decrease in functional capacity - the Duke Activity Status Index score was 4 points. According to the American Society of Anesthesiologists was grade IV. The patient's informed consent was obtained according to the guidelines of the local medical ethics committee.

\footnotetext{
Address for Correspondence: Dr. Todor Miroslavov Popov, Department of Otolaryngology, Medical University of Sofia, Sofia, Bulgaria

Phone: +359889987877_e-mail: popov@todorpopov.com - tmpopov@abv.bg

Received: 30 March 2016 Accepted: 22 June 2016 • DOI: 10.4274/balkanmedj.2016.0108

Available at www.balkanmedicaljournal.org

Cite this article as:

Popov TM, Marinov T, Rangachev J, Konov D, Belitova M. Cricotracheal Resection in a Patient with Severe Subglottic Stenosis - Advantages of a Temporary Non-

Cannulated Tracheostomy. Balkan Med J 2017;34:165-7

${ }^{\circ}$ Copyright 2017 by Trakya University Faculty of Medicine / The Balkan Medical Journal published by Galenos Publishing House.
} 


\section{Operative procedure and postoperative period with a temporary non-cannulated tracheostomy}

General anesthesia was induced and patient was ventilated through a laryngeal mask airway. Low collar incision was performed and the subplatysmal flap was raised. The trachea and cricoid cartilage were dissected from the soft tissues surrounding them, while protecting the recurrent nerve. The first two tracheal rings were shrunk, with a loss of cartilaginous support. Through an incision just beneath the pathological section, the patient was intubated with a tube and afterwards the stenotic segment, the cricoid arch and the first two tracheal rings were resected. The lumen of the stenosis was $2.5 \mathrm{~mm}$ (Figure 2). Severe adhesions were found and resected posteriorly enveloping the corresponding segment of the esophagus. Afterwards, the trachea was released from the upper mediastinum and thyrohyoid release of the larynx was performed. Thyrotracheal anastomosis was performed using

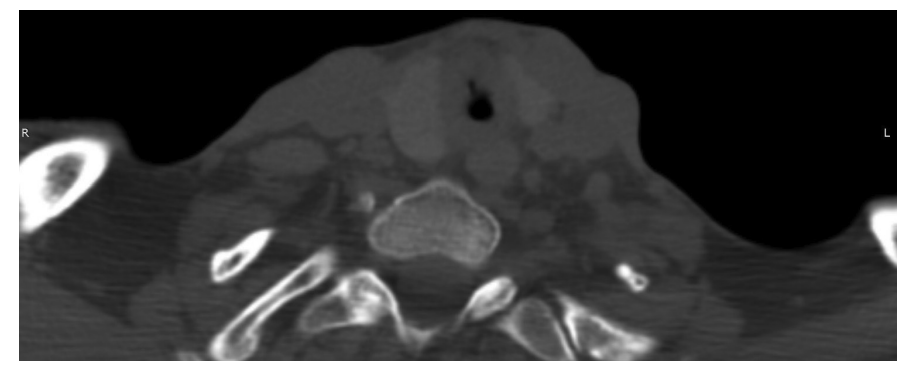

FIG. 1. Axial computer tomography slice showing the subglottic stenosis.

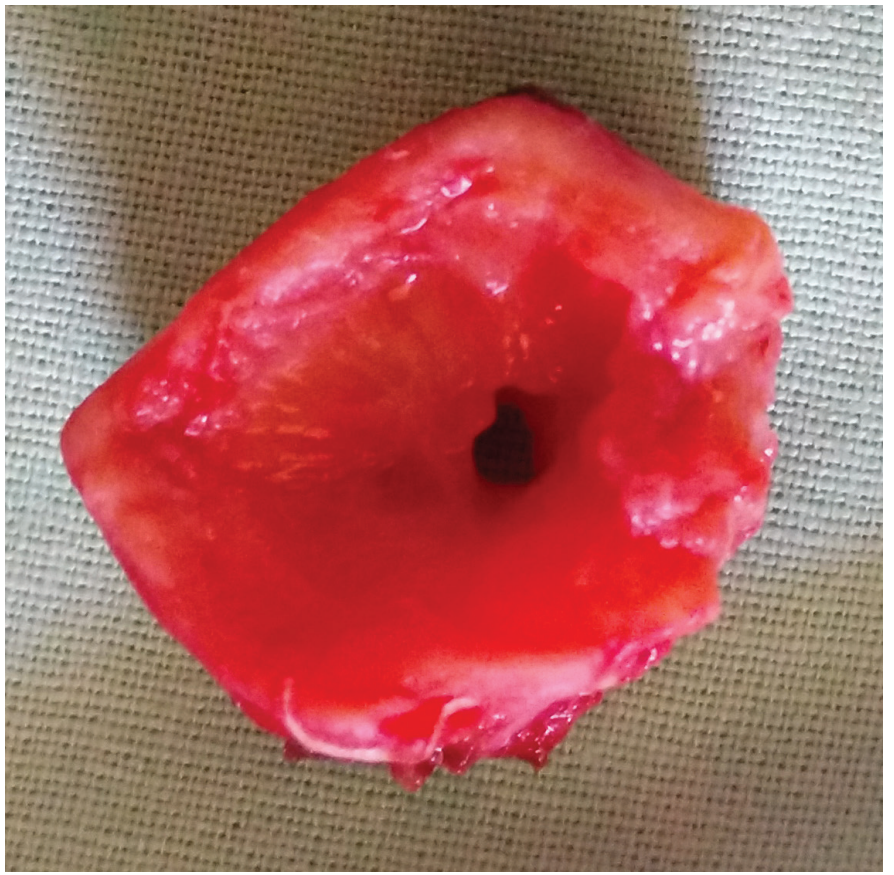

FIG. 2. Post-operative photograph of the tracheal segment of the stenosis with a lumen of $2.5 \mathrm{~mm}$.
MonoPlus ${ }^{\circledR}$ (B. Braun, Melsungen, Germany) (polydioxanone) sutures; the latter was verified for air leaks via laryngeal mask ventilation. At the level of the collar incision (approximately $4 \mathrm{~cm}$ beneath the anastomosis), an intercartilaginous incision of the trachea was performed and both superior and inferior skin flaps were sutured with the tracheal edges creating a tracheostomy, as shown in Figure 3, without placing a cannula. The postoperative period was uneventful. Crusts were removed meticulously from the tracheostomy and the latter was closed under local anesthesia at the end of the first month. One additional finding of interest was a significant post-operative increase in the ejection fraction up to $41 \%$. The follow-up period was one year and no signs of restenosis or notable granulation were registered in the area of anastomosis or at the level of the temporary tracheostomy.

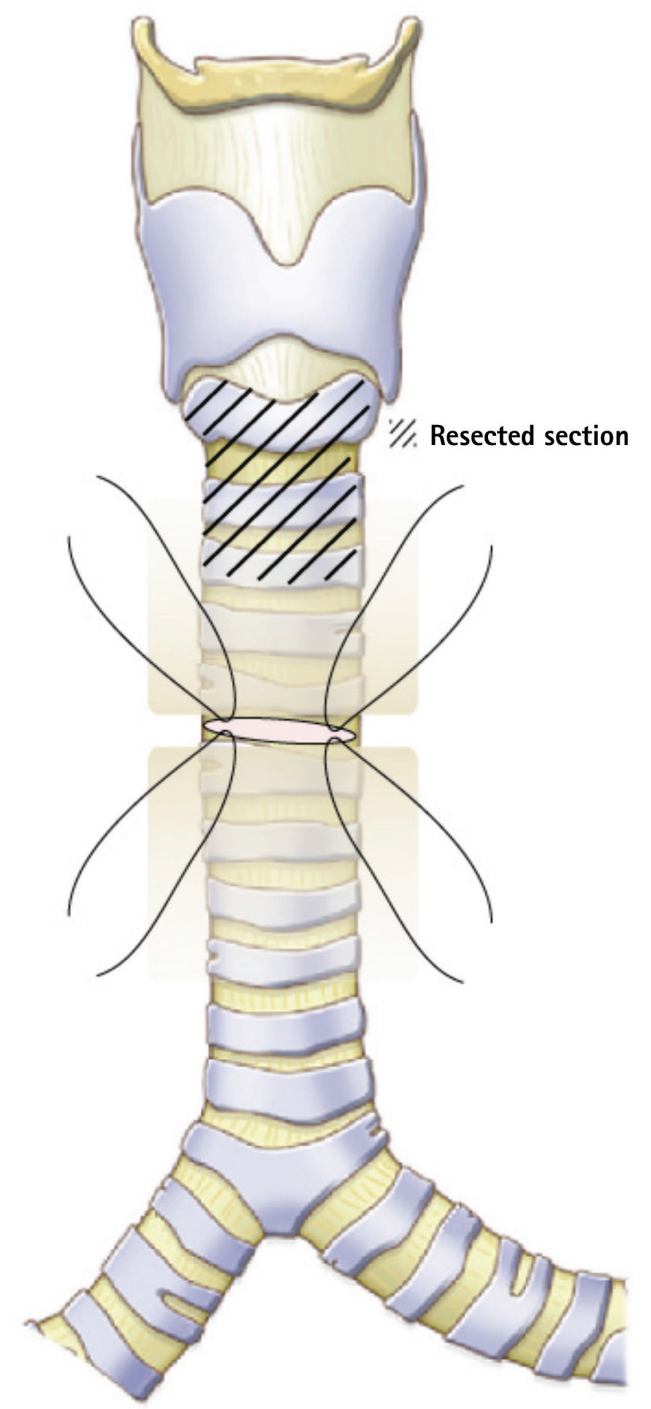

FIG. 3. Creating a temporary non-cannulated tracheostomy. Schematic illustration. 


\section{DISCUSSION}

Cricotracheal resection with primary anastomosis is an established effective treatment for subglottic and tracheal stenosis, with a reasonable success rate. Despite this, results in the published literature show a significant percentage of revision surgery and definitely not negligible unsuccessful rate in terms of decannulation. Nakache et al. (5) achieved the primary goal in $68 \%$ of cases as the decannulation rate increased to $88.5 \%$ after different types of revision surgery. Similar results are seen in the data published by Rubikas et al. (6), 86.3\%, and Deckard et al. (7), 86\%. Additionally, the group of Rubikas reported perioperative complications of $34.8 \%$ within 30 days of the operation in the group with cricotracheal resection.

By presenting this case, we would like to introduce a new option in cricotracheal resections - temporary non-cannulated tracheostomy. In our opinion, this addition to the classical surgical procedure gives the following advantages:

- Fixation of the trachea to the skin relieves any transitory tension on the anastomosis from the weight of the tracheobronchial tree.

- It acts as a valve which decreases the air-pressure in the upper airways during coughing and sneezing.

- It provides a very easy access point for video tracheoscopy of the anastomosis during the early post-operative period.

- Because of the lack of soft tissue between the tracheal edges and the skin flaps, placing a cannula, which could lead to secondary stenosis/granulations, is not necessary.

- There is no significant decrease in quality of life during the early post-op period since the patient breathes primarily through the upper airway and phonates almost normally.

- It acts as an easy emergency access point for ventilation if a disaster such as full anastomotic dehiscence occurs.

Negative aspects are the necessity of a second procedure to close the tracheostomy and the crusting of the stoma, which should be periodically cleaned in ambulatory settings. Prospective studies would be necessary to demonstrate the advantages of this technique.

Conflict of Interest: No conflict of interest was declared by the authors.

\section{REFERENCES}

1. Herrington HC, Weber SM, Andersen PE. Modern management of laryngotracheal stenosis. Laryngoscope 2006;116:1553-7.

2. Grillo HC. Development of tracheal surgery: a historical review. Part 1: techniques of tracheal surgery. Ann Thorac Surg 2003;75:610-9.

3. Bauer TL, Steiner KV. Virtual bronchoscopy: clinical applications and limitations. Surg Oncol Clin N Am 2007;16:323-8.

4. Taha MS, Mostafa BE, Fahmy M, Ghaffar MK, Ghany EA. Spiral CT virtual bronchoscopy with multiplanar reformatting in the evaluation of postintubation tracheal stenosis: comparison between endoscopic, radiological and surgical findings. Eur Arch Otorhinolaryngol 2009;266:863-6.

5. Nakache G, Primov-Fever A, Alon EE, Wolf M. Predicting outcome in tracheal and cricotracheal segmental resection. Eur Arch Otorhinolaryngol 2015;272:1471-5.

6. Rubikas R, Matukaityte I, Jelisiejevas JJ, Rackauskas M. Surgical treatment of non-malignant laryngotracheal stenosis. Eur Arch Otorhinolaryngol 2014;271:2481-7.

7. Deckard N, Yeh J, Soares DJ, Criddle M, Stachler R, Coticchia J. Utility of two-stage laryngotracheal reconstruction in the management of subglottic stenosis in adults. Ann Otol Rhinol Laryngol 2013;122:322-9. 